(C) 2010. This manuscript version is made available under the CC-BY-NC-ND 4.0 license http:// creativecommons.org/licenses/by-nc-nd/4.0/

\title{
On the use of the Bingham statistical distribution in microsphere-based constitutive models for arterial tissue
}

\author{
V. Alastrué*, P. Sáez*, M. A. Martínez*, M. Doblaré* \\ Group of Structural Mechanics and Materials Modelling (GEMM). \\ Aragón Institute of Engineering Research (I3A). Universidad de Zaragoza. \\ María de Luna, 3. E-50018 Zaragoza, Spain. \\ Centro de Investigación Biomédica en Red en Bioingeniería, Biomateriales y Nanomedicina (CIBER-BBN), \\ Aragon Health Sciences Institute, Spain.
}

\begin{abstract}
Constitutive models for arterial tissue have been an active research field during the last years. The main micro-constituents of blood vessels are different types of cells and the extra-cellular matrix formed by an isotropic high water content ground substance and a network composed of elastin and collagen fibres. Usually the arterial tissue has been modelled as a hyperelastic material within the framework of continuum mechanics, whereas inclusion of structural tensors into constitutive laws is the most widely used technique to introduce the anisotropy induced by the fibres. Though the different existing fibre bundles present a clear preferential direction, the dispersion inherent to biological tissue advices using of constitutive models including representative structural information associated to the spatial probabilistic distribution of the fibres. Lately, microsphere-based models have demonstrated to be a powerful tool to incorporate this information. The fibre dispersion is incorporated by means of an Orientation Density Function (ODF) that weights the contribution of each fibre in each direction of the micro-sphere. In previous works the rotationally symmetric von Mises ODF was successfully applied to the modelling of blood vessels. In this study the inclusion of the Bingham ODF into microsphere-based model is analysed. This ODF exhibits some advantages with respect to the von Mises one, like a greater versatility and a comparable response to uniaxial and equibiaxial tension tests.
\end{abstract}

Keywords: Bingham distribution, microsphere model, orientation density function, parameter identification, blood vessel

\section{Introduction}

A great number of constitutive laws have been recently proposed for modelling the mechanical behaviour of soft biological tissues. In general, this kind of tissues is composed of an extra-cellular matrix, mainly composed of an isotropic ground substance with a high water content in which a network of fibres - mainly composed of elastin, different kinds of collagen and proteoglycans - is embedded. The combined contribution of these constituents determines the mechanical response of the tissue, which turns out to be highly non-linear. These fibres are also responsible for the anisotropic response of the tissue, due to the existence of clear preferential orientations of the fibre bundles [20,17]. The accurate incorporation of these principal directions of anisotropy into the constitutive laws, as well as the bundle directional dispersion with

\footnotetext{
${ }^{*}$ Corresponding author. Tel.: +34976761912; Fax: +34976762578

Email address: victorav@unizar.es (V. Alastrué)
} 
respect to this preferential orientation is one of the challenges for modelling the mechanical behaviour of soft tissues.

Firstly developed anisotropic constitutive models for blood vessels were purely phenomenological. Fung et al. [8] proposed an exponential law of the deformations in the principal cylindrical directions in order to account for the anisotropic behaviour of the tissue. However, the incorporation of structural tensors into constitutive laws is nowadays the most widespread technique for the inclusion of anisotropy along deterministic preferential orientations [21, 14]. Later, directional fibre dispersion was introduced in the models by means of different methodologies. In Holzapfel et al. [15], a phenomenological parameter served to account for dispersion, whereas Gasser et al. [9] used the von Mises Orientation Density Function (ODF) [6] to determine a structural tensor representing the fibre distribution. More recently, models including fibre dispersion from a micro-structurally-based approach have been proposed [1].

Different results presented in previous works have shown that the incorporation of information relative to the directional dispersion of the micro-structural components seems to be an excellent choice to deal with the variability inherent to the mechanical behaviour of soft tissues [1]. This methodology, together with the computational homogenisation technique based on the integration of the microscopic contributions over the surface of the unit sphere - or rather the micro-sphere approach-, has revealed to be effective for the implementation of these models within a numerical framework. Considering the isotropic case, Miehe and co-workers presented a series of papers modelling damage and viscoelasticity in polymers [18, 10]. Nevertheless, not much work has been done in the numerical study of this approach accounting for anisotropy. Alastrue et al. [1] studied the validity of several discretisations for the integration of the von Mises ODF with application to blood vessels modelling.

This work focuses on the use of the Bingham ODF for the incorporation of anisotropy in the microsphere-based models, with application to the modelling of soft biological tissues. This function increases the versatility of this kind of models since it is capable of producing a wide variety of distributions among which the von Mises distribution is a particular case. One of the main advantages of the Bingham ODF is to consider three different concentration parameters in three orthogonal directions of the space. These orientations can be easily correlated with the three main directions of a blood vessel: circumferential, radial and axial.

Two different constitutive behaviour laws were used to model the micro-fibre mechanical response, namely, an exponential one and the transversely isotropic version of the eight-chain model [4]. The remainder of the paper is organised as follows: Section 2 comprises a brief description of the Bingham ODF. The constitutive model and the micro-fibres behaviour are addressed is Section 3, whereas Section 4 deals with the numerical treatment of the stress tensors. The parameter identification procedure used to adjust curves from simple tension tests on human coronary arteries and a comparison between both ODFs for homogeneous deformation are presented in Section 5 and, finally, Section 6 resumes some conclusions and final remarks.

\section{The Bingham distribution}

The Bingham ODF [5] was used to account for the dispersion of the collagen fibrils with respect to their preferential orientation. That function is expressed as

$$
\rho(\boldsymbol{r} ; \boldsymbol{A}) \frac{\mathrm{d} A}{4 \pi}=[K(\boldsymbol{A})]^{-1} \exp \left(\boldsymbol{r}^{\mathrm{t}} \cdot \boldsymbol{A} \cdot \boldsymbol{r}\right) \frac{\mathrm{d} A}{4 \pi},
$$

where $\boldsymbol{A}$ is a symmetric $3 \times 3$ matrix, $\mathrm{d} A$ is the Lebesgue invariant measure on the unit sphere, $\boldsymbol{r} \in \mathbb{U}^{2}$ and $K(\boldsymbol{A})$ is a normalizing constant. As its main features, it is worth noting that this distribution always exhibits antipodal symmetry, but not rotational symmetry for the general case. 


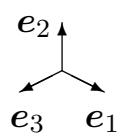

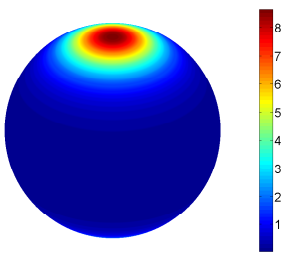

(a) $\kappa_{2}=0.0, \kappa_{3}=5.0$.

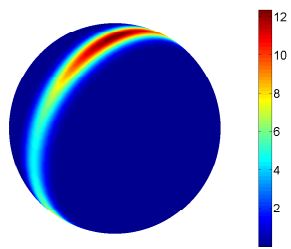

(d) $\kappa_{2}=49.0, \kappa_{3}=50.0$.

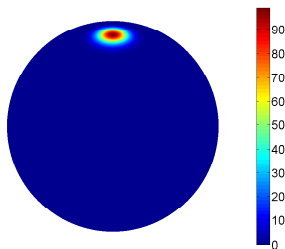

(b) $\kappa_{2}=0.0, \kappa_{3}=50.0$

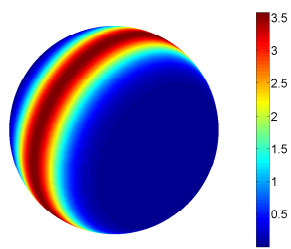

(e) $\kappa_{2}=10.0, \kappa_{3}=10.0$.

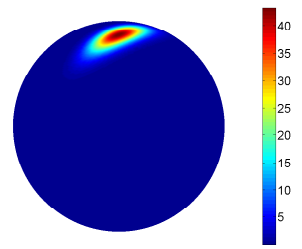

(c) $\kappa_{2}=40.0, \kappa_{3}=50.0$.

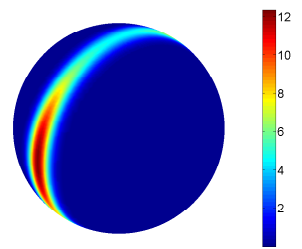

(f) $\kappa_{2}=50.0, \kappa_{3}=49.0$.

Figure 1: Representation of the Bingham ODF for different sets of parameters with $\kappa_{1}=0.0$.

Applying straightforward transformations, Eq. (1) can be rewritten as

$$
\rho(\boldsymbol{r} ; \boldsymbol{Z}, \boldsymbol{Q}) \frac{\mathrm{d} A}{4 \pi}=\left[F_{000}(\boldsymbol{Z})\right]^{-1} \operatorname{etr}\left(\boldsymbol{Z} \cdot \boldsymbol{M}^{\mathrm{t}} \cdot \boldsymbol{r} \cdot \boldsymbol{r}^{\mathrm{t}} \cdot \boldsymbol{Q}\right) \frac{\mathrm{d} A}{4 \pi},
$$

where $\operatorname{etr}(\bullet) \equiv \exp (\operatorname{tr}(\bullet)), \boldsymbol{Z}$ is a diagonal matrix with eigenvalues $\kappa_{1,2,3}, \boldsymbol{Q} \in \mathbb{Q}^{3}$ such that $\boldsymbol{A}=$ $\boldsymbol{Q} \cdot \boldsymbol{Z} \cdot \boldsymbol{Q}^{\mathrm{T}}$ and $F_{000}(\boldsymbol{Z})$ may be written as

$$
F_{000}(\boldsymbol{Z})=[4 \pi]^{-1} \int_{\mathbb{U}^{2}} \operatorname{etr}\left(\boldsymbol{Z} \cdot \boldsymbol{r} \cdot \boldsymbol{r}^{\mathrm{t}}\right) \mathrm{d} A={ }_{1} F_{1}\left(\frac{1}{2} ; \frac{3}{2} ; \boldsymbol{Z}\right),
$$

with ${ }_{1} F_{1}$ a confluent hypergeometric function of matrix argument as defined by [12].

Thus, the probability concentration is controlled by the eigenvalues of $\boldsymbol{Z}$, which might be interpreted as concentration parameters. Specifically, the difference between pairs of $\kappa_{1,2,3}-$ i.e., $\left[\kappa_{1}-\kappa_{2}\right]$, $\left[\kappa_{1}-\kappa_{3}\right]$ and $\left[\kappa_{2}-\kappa_{3}\right]-$ determines the shape of the distribution over the surface of the unit sphere. Therefore, the value of one of these three parameters may be fixed to a constant value without reducing the versatility of (2). Figure 1 shows different distributions of the fibre bundles concentration achieved for a constant value of $\kappa_{1}$ and varying values of $\kappa_{2}$ and $\kappa_{3}$. With this assumption, the distribution can expand along the plane composed of the associated direction with $\kappa_{2}$ and $\kappa_{3}$. As seen in Figure 1(a) and (b), setting two of the parameters equal to zero the Von Mises ODF is obtained. The highest value of the parameters is associated to the preferential orientation as show in Figure 1(c) and (f). When two parameters come close up, a rotational symmetry is achieved.

\section{Constitutive model}

Within the framework of continuum mechanics and considering the hypotheses of hyperelasticity, the strain energy density function used to model the arterial tissue is expressed as the sum of volumetric and isochoric parts (4), describing the strain energy associated to volume changes and purely isochoric deformations, respectively [7].

$$
\Psi=\Psi_{\text {vol }}+\Psi_{\text {ich }}
$$


The volumetric contribution is chosen as $\psi_{\text {vol }}=\frac{1}{D}[J-1]^{2}$, in order to obtain a quasi-incompressible behaviour depending on the value of the constant $D$ and the determinant of the deformation gradient tensor $J=\operatorname{det}(\boldsymbol{F})$.

Regarding the isochoric contribution, it is assumed to be a function of the isochoric deformation gradient tensor, namely, $\overline{\boldsymbol{F}}=J^{-1 / 3} \boldsymbol{F}$. A further decomposition into isotropic and anisotropic parts representing the contributions associated to the isotropic ground substance and to the collagen fibres embedded, renders

$$
\Psi_{\text {ich }}=\Psi_{\text {iso }}+\Psi_{\text {ani }} \text {. }
$$

A simple neo-Hookean term is chosen to model the former contribution, namely $\Psi_{\text {iso }}=$ $\mu\left[\operatorname{tr}\left(\overline{\boldsymbol{F}}^{\mathrm{t}} \cdot \overline{\boldsymbol{F}}\right)-3\right]$, whereas the micro-sphere approach is used to account for the collagen anisotropic contribution. Specifically, the collagen fibre contribution is modelled as

$$
\Psi_{\text {ani }}=\sum_{j=1}^{N} \Psi_{\mathrm{f}}^{j}=\sum_{j=1}^{N}\left[\sum_{i=1}^{m} \rho\left(\boldsymbol{r}^{i} ; \boldsymbol{Z}, \boldsymbol{Q}\right) \Psi_{\mathrm{f}}^{i}(\bar{\lambda})\right]^{j},
$$

where $\mathrm{N}$ is the number of anisotropy directions or rather fibre families (assumed to be one in this work), $\boldsymbol{r}^{i}$ are referential unit vectors associated with discretisation on the micro-sphere, and $\Psi_{\mathrm{f}}^{i}(\bar{\lambda})$ is the microscopic strain energy associated to the isochoric fibre stretch along the $\boldsymbol{r}^{i}$ direction, i.e., $\bar{\lambda}_{\mathrm{i}}=J^{-1 / 3}\left\|\boldsymbol{F} \cdot \boldsymbol{r}^{i}\right\|$. Then, (6) can be expressed as the continuous average denoted by $\langle(\bullet)\rangle$ as

$$
\Psi_{\text {ani }}=\left\langle n \rho \Psi_{\mathrm{f}}(\bar{\lambda})\right\rangle
$$

where $n$ is a constant representing as the isotropic network chain density [19]. With this formulation at hand, the average of the microscopic strain energies for a continuous space is defined as

$$
\left\langle n \rho \Psi_{\mathrm{f}}(\bar{\lambda})\right\rangle=\frac{1}{4 \pi} \int_{\mathbb{U}^{2}} n \rho \Psi_{\mathrm{f}} \mathrm{d} A .
$$

Based on this expression, the mechanical contribution of the collagen fibres to the macroscopic Kirchhoff stresses can be written as the continuous average including the ODF, namely

$$
\boldsymbol{\tau}=\left\langle n \rho \Psi_{\mathrm{f}}^{\prime} \bar{\lambda}^{-1} \overline{\boldsymbol{t}} \otimes \overline{\boldsymbol{t}}\right\rangle,
$$

where $\Psi_{\mathrm{f}}^{\prime}$ represents the derivative of the microscopic strain energy with respect to $\bar{\lambda}$, and $\overline{\boldsymbol{t}}=\overline{\boldsymbol{F}} \cdot \boldsymbol{r}$.

\subsection{Mechanical behaviour of the micro-fibres}

Two microscopic strain energy density functions were used to model the contribution of the collagen fibres to the macroscopic mechanical quantities [1]. First, a phenomenological exponential function frequently used to model the fibre response from a macroscopic phenomenological approach [14], namely

$$
n \Psi_{\mathrm{f}}^{i}\left(\bar{\lambda}_{i}\right)=\left\{\begin{array}{lll}
0 & \text { if } & \bar{\lambda}_{i}<1 \\
\frac{k_{1}}{2 k_{2}}\left[\exp \left(k_{2}\left[\bar{\lambda}_{i}^{2}-1\right]^{2}\right)-1\right] & \text { if } & \bar{\lambda}_{i} \geq 1
\end{array}\right.
$$

where $k_{1}$ is a stress-valued constant, $k_{2}$ is a dimensionless parameter, and $\bar{\lambda}_{i}$ denotes the isochoric stretch in the fibre direction of $\boldsymbol{r}^{i}$, i.e., $\bar{\lambda}_{i}=\left\|\overline{\boldsymbol{t}}^{i}\right\|$.

The particularisation of the eight-chain model [4] to the transversely isotropic case [16, 1] was also used to model microfibre contributions, i.e.:

$$
n \Psi_{\mathrm{f}}^{i}\left(\bar{\lambda}_{i}\right)=\left\{\begin{array}{ccc}
0 & \text { if } \quad \bar{\lambda}_{i}<1 \\
\frac{n K \Theta L}{4 A}\left[2 \frac{\bar{r}_{i}^{2}}{L^{2}}+\frac{1}{1-\bar{r}_{i} / L}-\frac{\bar{r}_{i}}{L}\right. & \text { if } \quad \bar{\lambda}_{i} \geq 1 \\
\left.-\frac{\ln \left(\bar{\lambda}_{i}^{4} r_{0}^{2}\right.}{4 r_{0} L}\left[4 \frac{r_{0}}{L}+\frac{1}{\left[1-r_{0} / L\right]^{2}}-1\right]-\Psi_{\mathrm{r}}\right] &
\end{array}\right.
$$


with $\bar{r}_{i}=\bar{\lambda}_{i} r_{0}$, and

$$
\Psi_{\mathrm{r}}=2 \frac{r_{0}^{2}}{L^{2}}+\frac{1}{1-r_{0} / L}-\frac{r_{0}}{L}
$$

being a repository constant accounting for a zero strain energy at $\bar{\lambda}_{i}=1$ [3]. Notice that in both cases fibres are assumed not to bear any load under compression.

\section{Numerical integration over the surface of the unit sphere}

The discretisation of the continuous orientation distribution on the unit sphere $\mathbb{U}^{2}$ is achieved by means of $m$ discrete orientation vectors $\left\{\boldsymbol{r}^{i}\right\}_{i=1, \cdots, m}$ and the corresponding weighting factors denoted $\left\{w^{i}\right\}_{i=1, \cdots, m}$. Accordingly, the continuous average introduced in Eq. (8) can be approximated by

$$
\langle(\bullet)\rangle=\frac{1}{4 \pi} \int_{\mathbb{U}^{2}}(\bullet) \mathrm{d} A \approx \sum_{i=1}^{m} w^{i}(\bullet)^{i} .
$$

In order to avoid accuracy loss due to the numerical approximation in (13), the 600 hundred integration directions discretisation of 39th degree proposed by $\mathrm{Heo}$ and $\mathrm{Xu}$ [11] was used to approximate the stress tensor and the normalisation constant $F_{000}$ in (3).

\section{Fitting human coronary artery experimental curves}

The strain energy functions in Section (3) was used to fit experimental curves of simple tension tests carried out on human coronary arteries [15]. Data coming from thirteen individuals for media and adventitia layers were fit. To be specific, experiments on samples along the circumferential and longitudinal directions of the cylindrical frame $\left\{\boldsymbol{e}_{\theta, z, r}\right\}$ were considered. A least-square scheme was used to minimise the functional

$$
\chi^{2}=\sum_{j=1}^{p}\left[\left[\sigma_{\theta \theta}-\sigma_{\theta \theta}^{\Psi}\right]_{j}^{2}+\left[\sigma_{z z}-\sigma_{z z}^{\Psi}\right]_{j}^{2}\right],
$$

with $p$ the number of experimental measures, $\sigma_{\theta \theta}$ and $\sigma_{z z}$ the Cauchy stress data obtained from the tests, and $\sigma_{\theta \theta}^{\Psi}$ and $\sigma_{z z}^{\Psi}$ representing the Cauchy stresses obtained from (9) as $\sigma^{\Psi}=J^{-1} \tau^{\Psi}$.

A single ODF was used to reproduce the curves for which the eigenvalues of the $Z$ matrix - namely, $\kappa_{1,2,3}$ - were assumed to be in the interval $[0, \infty)$ representing the concentration parameters associated to the radial, the longitudinal and the circumferential directions, respectively. Then, following experimental evidences [20], the preferential orientation of the microfibres were assumed to be contained in the plane formed by vectors $\boldsymbol{e}_{\theta}$ and $\boldsymbol{e}_{z}$ by fixing $\kappa_{1}=0$.

A quasi-Newton minimisation algorithm was used to minimise Eq. (14). The normalised root mean square error

$$
\varepsilon=\frac{1}{v} \sqrt{\chi^{2} /[p-q]}
$$

was used as a measure of the quality of the approximated set of parameters, where $q$ is the number of parameters to be identified so that $p-q$ is the number of degrees of freedom, and $v$ represents the mean stress defined as

$$
v=\frac{1}{p} \sum_{j=1}^{p}\left[\sigma_{\theta \theta}+\sigma_{z z}\right]_{j}
$$

Following Alastrué et al. [1], the deformation gradient tensors causing the stress-strain curves reported by Holzapfel et al. [15] were assumed to correspond to pure shear deformations, namely $\boldsymbol{F}=\lambda \boldsymbol{e}_{\theta} \otimes \boldsymbol{e}_{\theta}+$ $\lambda^{-1} \boldsymbol{e}_{z} \otimes \boldsymbol{e}_{z}+\boldsymbol{e}_{r} \otimes \boldsymbol{e}_{r}$ and $\boldsymbol{F}=\lambda^{-1} \boldsymbol{e}_{\theta} \otimes \boldsymbol{e}_{\theta}+\lambda \boldsymbol{e}_{z} \otimes \boldsymbol{e}_{z}+\boldsymbol{e}_{r} \otimes \boldsymbol{e}_{r}$ for the circumferential and longitudinal samples, respectively. 
Table 1: Material parameters identified based on exponential fibre behaviour.

\begin{tabular}{cccccccc}
\hline Layer & - & $\mu[\mathrm{kPa}]$ & $k_{1}[\mathrm{kPa}]$ & $k_{2}[-]$ & $\kappa_{2}[-]$ & $\kappa_{3}[-]$ & $\varepsilon[-]$ \\
\hline Adventitia & Mean & 7.560 & 55.176 & 14.755 & 63.534 & 57.334 & 0.186 \\
& SD & 4.658 & 29.106 & 8.638 & 16.301 & 14.355 & 0.072 \\
\hline Media & Mean & 1.268 & 19.622 & 3.437 & 61.606 & 63.416 & 0.072 \\
& SD & 0.634 & 7.867 & 0.810 & 19.420 & 19.635 & 0.029 \\
\hline
\end{tabular}

Table 2: Material parameters identified based on worm-like-chain fibre behaviour.

\begin{tabular}{ccccccccc}
\hline Layer & - & $\mu[\mathrm{kPa}]$ & $B[\mathrm{kPa}]$ & $r_{0}[\mathrm{~mm}]$ & $L[\mathrm{~mm}]$ & $\kappa_{2}[-]$ & $\kappa_{3}[-]$ & $\varepsilon[-]$ \\
\hline Adventitia & Mean & 7.560 & 3.622 & 0.752 & 1.000 & 61.210 & 57.830 & 0.273 \\
- & SD & 4.658 & 2.529 & 0.425 & 0.564 & 15.142 & 15.505 & 0.079 \\
\hline Media & Mean & 1.268 & 1.406 & 1.072 & 1.532 & 56.809 & 58.699 & 0.117 \\
- & SD & 0.634 & 0.527 & 0.496 & 0.705 & 17.311 & 17.911 & 0.058 \\
\hline
\end{tabular}

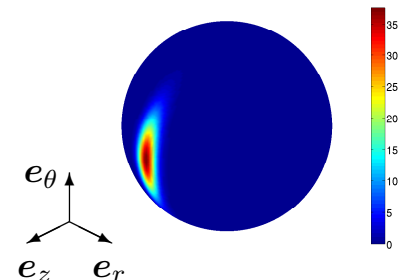

(a) Adventitia, exponential model: $\kappa_{2}=63.53, \kappa_{3}=$ 57.33 .

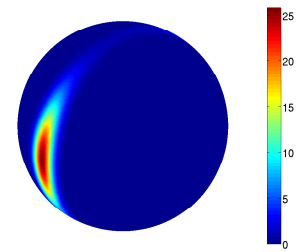

(b) Adventitia, worm-like- (c) Media, chain model: $\kappa_{2}=61.21$, model: $\kappa_{2}=61.61$ $\kappa_{3}=57.83$. $\quad \kappa_{3}=63.42$

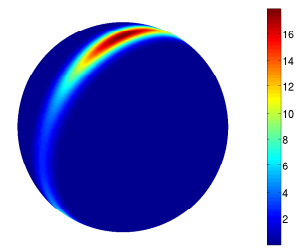

(d) Media, worm-like-chain model: $\kappa_{2}=56.81, \kappa_{3}=$ 58.70 .

Figure 2: Representation of the ODF for the identified averaged values $\kappa_{2}$ and $\kappa_{3}$ - the circumferential direction is referred to the $\boldsymbol{e}_{z}$ direction, whereas $\boldsymbol{e}_{x}$ corresponds to the longitudinal one.

The obtained sets of constant for each of the individuals together with the averaged values and the standard deviation are collected in Table 1 for the adventitia and media layers with exponential microstructural behaviour in (10) and, in the same way, in Table 2 for the worm-like structural behaviour, Eq. (11).

Three-dimensional plots of the Bingham ODF for the identified eigenvalues of $\boldsymbol{Z}$ are depicted in Figure 2. Note the similar values and shapes of the ODF rendered by the identified set of constants for both microstructural behaviours. As it is seen in Fig. 2(a) and (b), the fibres are preferentially oriented along the longitudinal direction in the adventitia layer, whereas its preferred orientation is along the circumferential direction in the medial layer, Fig. 2(c) and (d).

\section{Discussion}

The orientation of the micro-structural constituents of the blood vessels follow an inherent variability, but this dispersion is usually different respect to the three main intrinsic directions of the vessel: Circumferential, radial and axial. In Figure 3a the ellipsoidal shape of the SMC nucleus indicates a preferential circumferential direction, while in Figure $3 \mathrm{~b}$ a circular shape can be observed. Different optical techniques allow to quantify this dispersion parameters. Wicker2008 used a non-linear optical microscopy to quantify the fibre dispersion in rabbits basilar arteries, showing a change from circumferential to axil directions through the wall thickness. The Bingham ODF has been considered in this paper for the incorporation of 


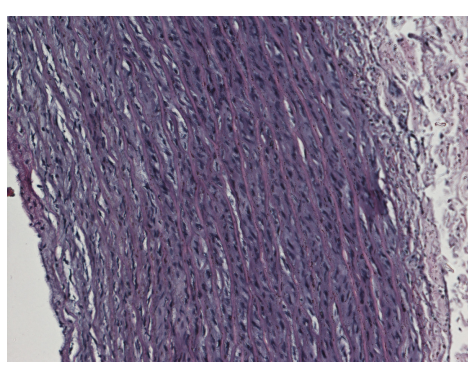

(a) Sample oriented in the circumferential plane.

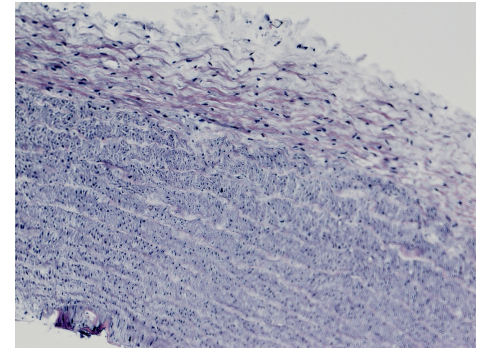

(b) Sample oriented in the axial plane.

Figure 3: Microphotographies of the histological pig carotid samples.

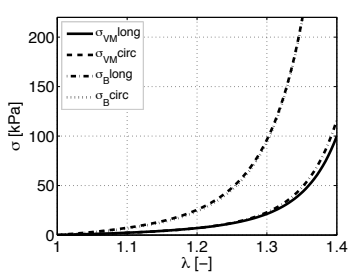

(a) Uniaxial test, media.

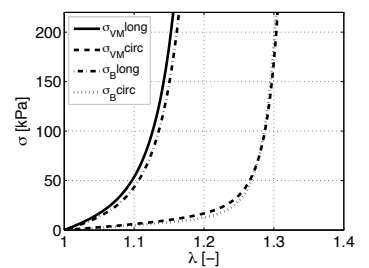

(b) Uniaxial test, adventitia.

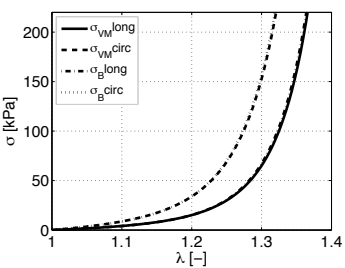

(c) Biaxial test, media.

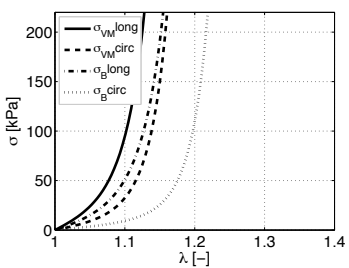

(d) Biaxial test, adventitia.

Figure 4: Comparison between von Mises and Bingham ODF for exponential fibre behaviour (Media and adventitia layer).

anisotropy to a hyperelastic micro-sphere-based constitutive law with application to the modelling of the vascular tissue. This ODF allows to include point-wise different dispersion parameters in each principal direction of the blood vessel, as represented in Figure 1.

The Bingham ODF was implemented within a numerical framework. The obtained results show a good agreement with the experimental data, with values of the normalised root means squared error very similar to those reported by Alastrué et al. [1] coming from fittings of the same experimental data.

Homogeneous deformation states were reproduced in order to compare both the von Mises and Bingham ODFs. An equivalent response is obtained for the two ODFs in the uniaxial tests (Figure 4(a) and (b)). Not such a good agreement was obtained for the equibiaxial tests (Figure 4(c) and (d)) due to the fact that the model paramters were obtained from uniaxial tests and [13]. In view of these results, it is worth noting that the use of the Bingham distribution allowed getting similar results with a single family of fibres instead of the two helically oriented families of fibres modelled by means of the von Mises ODF. This fact, which is crucial in the reduction of the computation associated the numerical calculation of the macroscopic stress, points out the feasibility of the Bingham distribution to include anisotropy in the micro-sphere based models. In addition, two Bingham ODFs might be incoroporated to the models in order to capture the fibre orientation in case the angle between both families was too wide to obtain a feasible distribution with a sigle Bingham ODF.

Nevertheless, some simplifications have been taken for the obtaining of the results here presented. The most important one regards the use of the eigenvalues of $Z$ as additional identification parameters for macroscopic stress-stretch curves. On the contrary, those parameters should by identified from measurements at the micro-structural level [17]. Moreover, the eigenvectors of $\boldsymbol{Z}$ were assumed to coincide with directions $\boldsymbol{e}_{\theta}, \boldsymbol{e}_{\theta}$ and $\boldsymbol{e}_{z}$, when the actual orientation at the micro-structural level can follow a more random distribution. However, this simplification is supported by strong experimental evidences, since most of the histological studies show that most of the fibres are comprised in that plane [20]. Moreover, the contribution of the isotropic neo-Hookean term constant $\mu$ was fixed to value obtained by Holzapfel et al. [15], which 


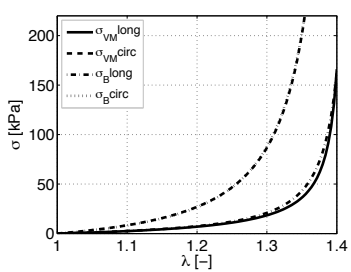

(a) Uniaxial test, media.

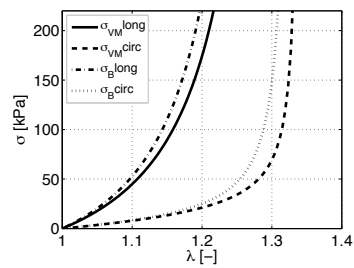

(b) Uniaxial test, adventitia.

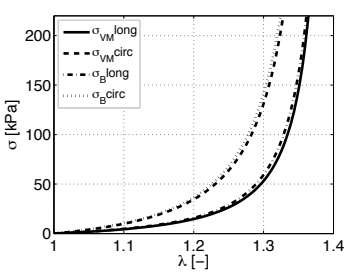

(c) Biaxial test, media.

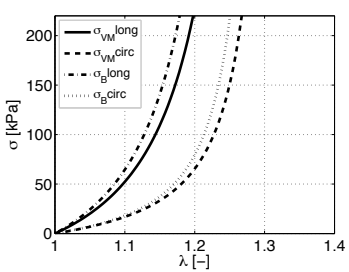

(d) Biaxial test, adventitia.

Figure 5: Comparison between von Mises and Bingham ODF for eight-chain model fibre behaviour (Media and adventitia layer).

might influence the results of the fittings. Nevertheless, it has been reported that the isotropic contribution to the macroscopic stress is only relevant up to certain value of stretch, from which collagen fibres bear most of the load [14]. In addition, fixing $\mu$ allowed us to establish direct comparisons with the values reported in Alastrué et al. [1]. Finally, the deformation gradient causing the stress-stretch curves was assumed to correspond to a uniaxial and equibiaxial tension with incompressibility constraint. Other deformation fields fulfilling this requirement might have been used, though previous studies show that the obtained constants are barely dependent on the used deformation field [1].

In summary, the presented results show the capability of the presented micro-sphere models together with the Bingham distribution to reproduce the mechanical behaviour of soft biological tissues, in particular, of the human coronary artery. Nevertheless, some points could be modified in order to improve the capabilities of the proposed models. Among them, the characterization and analysis of non-homogeneous deformation states and the performance of the numerical integration scheme is crucial for the generalization of the micro-sphere based models. Its accuracy has been shown to be dependent on the degree of anisotropy, for which a high order 600 integration directions discretisation was used here. It has been shown that the application of non-linear transformations can drastically reduce the number of necessary integration directions which, in turn, carries the reduction of the computational resources demand [2].

\section{Acknowledgements}

Financial support from the Spanish Ministry of Education and Science through research project DPI2007-63254 and Instituto de Salud Carlos III through the CIBER initiative is highly appreciated.

\section{Bibliography}

[1] Alastrué, V., Martínez, M., Doblaré, M., Menzel, A., Jan. 2009. Anisotropic micro-sphere-based finite elasticity applied to blood vessel modelling. J Mech Phys Solids 57 (1), 178-203.

[2] Alastrué, V., Martínez, M. A., Menzel, A., Doblaré, M., 2009. On the use of non-linear transformations for the evaluation of anisotropic rotationally symmetric directional integrals. application to the stress analysis in fibred soft tissues. Int J Numer Meth Eng 79 (4), 474-504.

[3] Alastrue, V., Rodriguez, J., Calvo, B., Doblare, M., Sep. 2007. Structural damage models for fibrous biological soft tissues. Int J Solids Struct 44 (18-19), 5894-5911.

[4] Arruda, E. M., Boyce, M. C., Feb. 1993. A three-dimensional constitutive model for the large stretch behavior of rubber elastic materials. J Mech Phys Solids 41 (2), 389-412.

[5] Bingham, C., 1974. An antipodally symmetric distribution on the sphere. Ann Stat 2 (6), 1201-1225. 
[6] Fisher, R., May 1953. Dispersion on a sphere. Proc R Soc Lon Ser-A 217, 295-305.

[7] Flory, P. J., 1961. Thermodynamic relations for high elastic materials. T Faraday Soc 57, 829-838.

[8] Fung, Y. C., Fronek, K., Patitucci, P., 1979. Pseudoelasticity of arteries and the choice of its mathematical expression. Am J Physiol Heart Circ Physiol 237 (5), H620-631.

[9] Gasser, T. C., Ogden, R. W., Holzapfel, G. A., 2006. Hyperelastic modelling of arterial layers with distributed collagen fibre orientations. J Roy Soc Interface 3, 15-35.

[10] Göktepe, S., Miehe, C., Oct. 2005. A micro-macro approach to rubber-like materials. part iii: The micro-sphere model of anisotropic mullins-type damage. J Mech Phys Solids 53 (10), 2259-2283.

[11] Heo, S., Xu, Y., 2001. Constructing fully symmetric cubature formulae for the sphere. Math Comput 70, 269-279.

[12] Herz, C. S., 1955. Bessel functions of matrix argument. Ann Math 61 (3), 474-523.

[13] Holzapfel, G. A., Jan. 2006. Determination of material models for arterial walls from uniaxial extension tests and histological structure. J Theor Biol 238 (2), 290-302.

[14] Holzapfel, G. A., Gasser, T. C., Ogden, R. W., Jul. 2000. A new constitutive framework for arterial wall mechanics and a comparative study of material models. J Elasticity V61 (1), 1-48.

[15] Holzapfel, G. A., Sommer, G., Gasser, C. T., Regitnig, P., Nov. 2005. Determination of layer-specific mechanical properties of human coronary arteries with nonatherosclerotic intimal thickening and related constitutive modeling. Am J Physiol Heart Circ Physiol 289 (5), H2048-2058.

[16] Kuhl, E., Menzel, A., Garikipati, K., 2006. On the convexity of transversely isotropic chain network models. Philos Mag 86, 3241-3258.

[17] Landuyt, M., 2006. Structural quantification of collagen fibers in Abdominal Aortic Aneurysms. Master's thesis, Royal Institute of Technology in Stockholm, Department of Solid Mechanics and Ghent University, Department of Civil Engineering.

[18] Miehe, C., Göktepe, S., Oct. 2005. A micro-macro approach to rubber-like materials. part ii: The micro-sphere model of finite rubber viscoelasticity. J Mech Phys Solids 53 (10), 2231-2258.

[19] Miehe, C., Göktepe, S., Lulei, F., Nov. 2004. A micro-macro approach to rubber-like materials-part i: the non-affine micro-sphere model of rubber elasticity. J Mech Phys Solids 52 (11), 2617-2660.

[20] Rhodin, J. A. G., 1980. Handbook of Physiology, The Cardiovascular System. Vol. 2. American Physiological Society, Bethesda, Maryland, Ch. Architecture of the vessel wall, pp. 1-31.

[21] Weiss, J. A., Maker, B. N., Govindjee, S., Aug. 1996. Finite element implementation of incompressible, transversely isotropic hyperelasticity. Comput Method Appl M 135 (1-2), 107-128. 